\title{
Identification, cross-taxon transferability and application of full-length cDNA SSR markers in Phyllostachys pubescens
}

\author{
Yuan Lin ${ }^{1}$, Jiang-Jie Lu', Miao-Dan Wu' ${ }^{1}$, Ming-Bing Zhou', Wei Fang ${ }^{1}$, Yuji Ide ${ }^{2}$ and Ding-Qin Tang ${ }^{1,2^{*}}$
}

\begin{abstract}
Current databases of Phyllostachys pubescens full-length cDNAs (FL-CDNAs) provide a rich source of sequences for the development of potential FL-cDNA simple sequence repeat (SSR) markers. We screened 10,608 P. pubescens cDNAs, discovering 1614 SSRs in 1382 SSR-containing FL-cDNAs. The SSRs were more abundant within transposable elements (TEs) than expressed sequence tags (ESTs) and genome survey sequences (GSSs), and specific dinucleotide repeats tended to associate with particular TE families: $(T A)_{n}$ with En/Spm and $(C T)_{n}$ with Mutator. A selected panel of $100 \mathrm{FL}-c D N A s$ containing type I SSRs yielded 68 functional SSR markers with an average polymorphism information content (PIC) value of 0.12, among which 22 loci contained polymorphisms. These markers became less transferrable $(83.1 \% \rightarrow 69.9 \% \rightarrow 49.3 \%)$ but more polymorphic $(79.4 \% \rightarrow 92.3 \% \rightarrow 92.8 \%)$ with increasing phylogenetic distance (intra-genus $\rightarrow$ intra-subtribe $\rightarrow$ intra-family). Transferability and polymorphism also depended on the location of the marker, with those located in the coding region being more transferrable (69.1\%) and less polymorphic (89.4\%) than those in the 5'-UTR (63.4\% transferable, 90.7\% polymorphic) and the 3'-UTR (61.8\% transferable, $91.4 \%$ polymorphic). As proof of principle, we were able to use our FL-CDNA SSR markers to identify the parental stocks in interspecific hybrids of bamboo within and beyond P. pubescens, and estimate the outcrossing rate for $P$. pubescens. Our research should facilitate molecular breeding in bamboo species where original genetic markers are scarce.
\end{abstract}

Keywords: Phyllostachys pubescens (edulis); Microsatellite (SSR); Cross-taxon transferability /polymorphism; Hybrid identification; Outcrossing-rate estimation

\section{Background}

Bambusoideae is a subfamily of the grass family Poaceae and is further divided into nine subtribes comprising more than 80 bamboo genera and about 1400 species worldwide. Fifty genera and more than 500 species are found in China, among which Phyllostachys pubescens (synonym: $P$. edulis) is commercially the most important species providing the third largest source of timber and the most predominant source of bamboo shoots. P. pubescens plantations cover an area of 3 million ha (approximately $2 \%$ of the total forest area), which has doubled over the last 30 years and taken on a more important ecological role (Fu 2001). Compounds extracted from

\footnotetext{
* Correspondence: tang@zafu.edu.cn

'The Nurturing Station for the State Key Laboratory of Subtropical

Silviculture, Zhejiang A \& F University, Zhejiang Province, LinAn 31300, P R China

${ }^{2}$ Laboratory of Forest Ecosystem Studies, The University of Tokyo, 1-1-1 Yayoi, Bunkyo-ku, Tokyo 113-8657, Japan
}

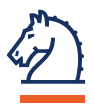

P. pubescens have recently shown potential for the treatment of obesity and other diseases (Higa et al. 2012). However, various problems associated with $P$. pubescens plantations including its simultaneous flowering intervals of more than 60 years and recovers from a limited number of clones (Janzen 1976; Watanabe et al. 1982). Additionally, the little knowledge of its basic biology, genetics and breeding system bring about the practical difficulties associated with the identification and characterization of superior genotypes.

Molecular markers developing from microsatellites, also known as simple sequence repeats (SSRs) with characterization of high genome coverage, random dispersion, co-dominant inheritance, reproducibility and amenability to automation in high throughout genotyping, have gained considerable spotlight recently. By now, microsatellite markers have been developed for several other bamboo species, e.g. six loci for Bambusa arundinacea 
(Nayak and Rout 2005), eight loci for Sasa senanensis (Miyazaki et al. 2009) and eight loci for S. cernua (Kitamura and Kawahara 2009). We identified 19 GenBank microsatellite markers in $P$. pubescens and related species (Tang et al. 2010), and 15 expressed sequence tag (EST) SSR markers for Bambusa species (Dong et al. 2011). Recently, the Bamboo Full-Length cDNA Project (Peng et al. 2010) has generated a vast amount of publicly-available $P$. pubescens cDNA sequence data that can be used for gene discovery, comparative genomics/transcriptomics and marker development. Microsatellites derived from cDNAs or ESTs are highly transferable to closely related species (Zhang et al. 2005) facilitating the development of gene-based maps that may increase the efficiency of marker-assisted selection through the use of candidate genes (Rossi et al. 2003; Lu et al. 2006).

Here, we report the use of $P$. pubescens full-length cDNA (FL-cDNA) sequences to 1) analyze the association between SSRs and transposable elements (TEs) in the transcriptome; 2) develop and validate FL-cDNA SSR markers and determine their transferability to other bamboo species; and 3) apply the polymorphic SSR markers to estimate outcrossing rates in $P$. pubescens and identify bamboo interspecies hybrids.

\section{Results and discussion}

\section{Association between SSRs and TEs in the $P$. pubescens} transcriptome

We analyzed 10,608 P. pubescens FL-cDNA sequences available in NCBI GenBank, representing $~ 7171 \mathrm{~kb}$ of DNA. EST-trimmer was used to remove poly $(\mathrm{A} / \mathrm{T})$ runs, and the remaining sequence data were screened using MISA, identifying 2330 SSRs in 2014 cDNAs, the remaining cDNAs lacking SSRs. The sequences were clustered with CAP3, reducing the collection to 1614 non-redundant SSRs in 1382 cDNA contigs (Additional file 1: Figure S1). Peng et al. (2010) described the distribution of SSRs in the P. pubescens transcriptome in detail. Therefore, we selectively analyzed the non-redundant cDNA sequences and contigs with RepeatMasker to determine the association between SSRs and TEs because previous reports have shown that many SSRs are located in TEs (Richard et al. 2008), e.g. 50\% of SSRs in the human genome (Scherer 2008), and that SSRs are closely associated with TEs in rice (Akagi et al. 2001; Temnykh et al. 2001) and barley (Wei et al. 2002). The results revealed 95 TEs, representing $13.52 \mathrm{~kb}(0.27 \%)$ of the total cDNA sequence data. Further analysis showed that 29 TEs contained a total of 39 SSRs, accounting for $822 \mathrm{bp}$ (6.41\%) of the total TE DNA sequences. In comparison, the nonredundant EST sequence data (7089 cDNAs refined from the original 10,608 sequences) contained 1614 SSRs, accounting for $2.60 \%$ of the total cDNA sequences in length. Therefore, SSRs were approximately 2.5-, 65.4-fold more abundant in TEs compared to cDNAs (Table 1) and whole genome $(0.098 \%$ based on the analysis of genome survey sequences (GSSs; Tang et al. 2010)). It is possible that SSRs within TEs are also involved in the regulation of gene expression (Tomilin 2008).

Some studies have also suggested associations between specific SSR motifs and particular TE families, e.g. (TA $)_{n}$ is often found in the $5^{\prime}$-UTR of Micron element transposase genes in rice (Akagi et al. 2001; Temnykh et al. 2001). We also investigated the distribution of SSRs among DNA transposons, and found they were most likely to occur in En/Spm elements $(33.87 \%$ of the total $E n / S p m$ DNA sequence). Six SSRs were found in five $E n / S p m$ elements, with one element containing two SSRs (Table 2). Mutator elements were the next most likely to contain SSRs (14.19\% of the total Mutator DNA sequence). Thirteen SSRs were found in 12 Mutator elements, again with one element containing two SSRs (Table 2). The situation was very different among retrotransposons, with only $0.30 \%$ of the total Ty1-copia DNA sequence and 0\% of Ty3-gypsy DNA sequence made up of SSRs. More detailed investigation of specific repeat motifs showed that four of the six SSRs found in En/Spm elements were TA/AT repeats, and 10 of the 13 SSRs found in Mutator elements were CT/AG repeats. All 13 of the Mutator SSRs and six of the En/Spm SSRs were located in the $5^{\prime}$-UTR. It has been reported that TE molecular markers (mPing) showed significantly higher levels of polymorphism than all other molecular markers in closely-related rice cultivars (Monden et al. 2009). Considering that it is difficult to detect genetic variation in $P$. pubescens using ordinary markers (Lin et al. 2009; Tang et al. 2010), SSRs in TEs therefore appear to be promising markers for bamboo species.

\section{Development and polymorphism assessment of FL-CDNA SSR markers for $P$. pubescens}

Original collection of 10,680 P. pubescens FL-cDNA sequences produced 1382 cDNA contigs containing SSRs. Sequences containing mononucleotide repeat motifs were excluded, leaving 1051 cDNA sequences containing SSRs with 2-6 nt repeats motifs (Additional file 1: Figure S1). Following the procedure already adopted for rice (Temnykh et al. 2001). We were able to design primer pairs for 583 (55\%) of these cDNAs, the remainder offering either insufficient flanking DNA (over half of the SSRs were found in the $5^{\prime}$ or $3^{\prime}$ UTRs) or flanking DNA that was unsuitable for primer design. Only 325 (24.1\%) of the SSRs were type I repeats (>20 bp), which offer greater potential for marker development. The 100 most promising sequences were selected for PCR validation, including dinucleotide repeats with $\geq 12$ repeat units, trinucleotide repeats with $\geq 8$ repeat units, tetranucleotide repeats with $\geq 6$ repeat units, pentanucleotide and 
Table 1 Association between FL-cDNA SSRs and transposable elements (TEs) in $P$. pubescens

\begin{tabular}{|c|c|c|c|c|c|c|c|c|c|c|c|}
\hline \multirow[t]{2}{*}{ TE family } & \multirow[t]{2}{*}{ No. } & \multirow{2}{*}{$\begin{array}{l}\text { Length } \\
\text { (bp) }\end{array}$} & \multirow{2}{*}{$\begin{array}{l}\text { No. of } \\
\text { TE-SSR }\end{array}$} & \multirow{2}{*}{$\begin{array}{l}\text { No. of } \\
\text { SSR-TE }\end{array}$} & \multirow{2}{*}{$\begin{array}{l}\text { TE-SSR/SSR-TE (\%) } \\
\text { (in length ) }\end{array}$} & \multicolumn{6}{|c|}{ No. of SSRs with repeat units of: } \\
\hline & & & & & & $1 \mathrm{nt}$ & $2 \mathrm{nt}$ & $3 \mathrm{nt}$ & $4 \mathrm{nt}$ & $5 \mathrm{nt}$ & $6 \mathrm{nt}$ \\
\hline Total TEs & 95 & 13522 & 36 & 29 & 6.08 & 1 & 31 & 4 & 0 & 1 & 0 \\
\hline En/Spm & 8 & 815 & 6 & 5 & 33.87 & 0 & 5 & 1 & 0 & 1 & 0 \\
\hline Mutator & 28 & 2931 & 13 & 12 & 14.19 & 1 & 10 & 2 & 0 & 0 & 0 \\
\hline Ty1-copia & 20 & 4010 & 1 & 1 & 0.3 & 0 & 0 & 1 & 0 & 0 & 0 \\
\hline Ty3-gyspy & 17 & 2913 & 0 & 0 & 0 & 0 & 0 & 0 & 0 & 0 & 0 \\
\hline Other TEs & 22 & 2853 & 16 & 11 & 8.70 & 0 & 16 & 0 & 0 & 0 & 0 \\
\hline EST & 7089 & 4942281 & 1614 & N.A. & 2.60 & 271 & 489 & 789 & 30 & 14 & 21 \\
\hline
\end{tabular}

hexanucleotide repeats with $\geq 5$ repeat units and some compound SSRs with $>24$ repeats (Table 3). We found that 32 of the selected cDNAs were unsuitable because the PCR failed to generate a product (four cDNAs) or generated products lacking SSRs ( 28 cDNAs), but the remaining 68 sequences allowed the development of FLcDNA SSR markers (Table 3). These contained 18 compound SSRs, 19 dinucleotide repeats, 18 trinucleotide repeats, four tetranucleotide repeats, three pentanucleotide repeats and six hexanucleotide repeats. Interestingly, although 45 of the cDNAs (66.2\%) generated the anticipated PCR product, 16 (23.5\%) generated products with more repeats than expected, five (7.4\%) generated

Table 2 Distribution of SSRs in En/Spm and Mutator transposons

\begin{tabular}{|c|c|c|c|c|c|}
\hline ID & SSR motifs & Length (bp) & Starting & Ending & Location \\
\hline \multicolumn{6}{|c|}{ SSR distribution in En/Spm transposons } \\
\hline FP091991 & $(\mathrm{GAGGA})_{6}$ & 30 & 109 & 138 & CDS \\
\hline FP091422 & $(\mathrm{TA})_{22}(\mathrm{CA})_{9}$ & 62 & 12 & 73 & $5^{\prime} U T R$ \\
\hline FP097776 & $(T A)_{23}$ & 46 & 1 & 46 & 5'UTR \\
\hline FP100462 & $(\mathrm{TA})_{31}$ & 62 & 14 & 75 & $5^{\prime} \cup T R$ \\
\hline FP100841 & $(\mathrm{CGG})_{6}$ & 18 & 38 & 55 & 5'UTR \\
\hline FP100858 & $(\mathrm{AT})_{29}$ & 58 & 22 & 79 & 5'UTR \\
\hline \multicolumn{6}{|c|}{ SSR distribution in Mutator transposons } \\
\hline FP100733 & $(\mathrm{TC})_{8}-(\mathrm{GGC})_{5}$ & 89 & 20 & 108 & 5'UTR \\
\hline FP100664 & $(A G)_{17}$ & 34 & 32 & 65 & 5'UTR \\
\hline FP094905 & $(\mathrm{CT})_{17}$ & 34 & 1 & 34 & $5^{\prime} \cup T R$ \\
\hline FP099988 & $(\mathrm{CT})_{19}$ & 38 & 1 & 38 & $5^{\prime} \cup T R$ \\
\hline FP094782 & $(\mathrm{CT})_{12}$ & 24 & 7 & 30 & 5'UTR \\
\hline FP099842 & $(\mathrm{CT})_{15}$ & 30 & 4 & 33 & $5^{\prime} \cup T R$ \\
\hline FP091749 & $(\mathrm{CT})_{23}$ & 46 & 2 & 47 & $5^{\prime}$ UTR \\
\hline FP093400 & $(G A)_{16}$ & 32 & 23 & 54 & $5^{\prime}$ UTR \\
\hline FP096707 & $(G A A)_{8}$ & 24 & 36 & 59 & 5'UTR \\
\hline FP096801 & $(T C)_{8}$ & 16 & 2 & 17 & $5^{\prime}$ UTR \\
\hline FP099127 & $(A G)_{18}$ & 36 & 40 & 75 & 5'UTR \\
\hline FP099725 & $(C)_{13}$ & 13 & 1 & 13 & 5'UTR \\
\hline
\end{tabular}

products with fewer repeats than expected, and two (PBM050 and PBM055) generated products with different repeats and flanking sequences than those anticipated. The unanticipated amplification resulted in three SSR markers (PBM036, PBM055 and PBM 077) containing type II repeats (12-19 bp in length) and one marker (PBM079) shorter than $12 \mathrm{bp}$. In total, 67 sequences were deposited in GenBank (accession nos GU644371-GU644438). Based on BLASTX analysis, putative functions were assigned to most $(66.2 \%)$ of the cDNA sequences with significant similarity to known proteins, whereas $27.9 \%$ matched unknown/hypothetical proteins and $5.9 \%$ were novel sequences (Table 3).

One hundred and seven primer pairs finally yielded 68 FL-cDNA SSR markers for $P$. pubescens, which is towards the lower end of the $60-90 \%$ success rate previously reported in sugarcane (Cordeiro et al. 2001), barley (Thiel et al. 2003), wheat ( $\mathrm{Yu}$ et al. 2004) and peanut (Liang et al. 2009). Squirrell et al. (2003) defined the successive loss of sequenced fragments and designed primers, until arriving at a final collection of "working SSRs" producing discrete bands of the expected size, as the "attrition rate". Kofler et al. (2008) reported a high attrition rate when developing SSR markers from enriched libraries, BAC-end sequences and ESTs in rye, possibly reflecting the large number of TEs in the rye genome. Tero et al. (2006) found that the number of SSR markers was reduced when the markers were predominantly located within TEs. Squirrell et al. (2003) suggested that SSR marker development would be challenging in polypoid species and species such as wheat and rye with large numbers of TEs. P. pubescens has $2 \mathrm{n}=48$ chromosomes and is thought to be tetraploid ( $\mathrm{Li}$ et al. 1999). The genome is > $2000 \mathrm{Mb}$, which is approximately 5.4 times larger than diploid cultivated rice (Gui et al. 2007), and it contains a large number of TEs (Zhong et al. 2010; Zhou et al. 2010a, b, c). The slightly higher attrition rate we encountered therefore seems reasonable when considering the chromosomal polyploidy, size and TE content of the genome. We also encountered a higher attrition rate in B. oldhamii (Li et al. 2001), a hexaploid bamboo 
Table 3 Characteristics of the $P$. pubescens SSR markers derived from FL-CDNAs

\begin{tabular}{|c|c|c|c|c|c|c|c|c|c|}
\hline \multirow[t]{2}{*}{ No. } & \multirow{2}{*}{$\begin{array}{l}\text { Marker } \\
\text { Name }\end{array}$} & \multicolumn{2}{|c|}{ Accession no. } & \multirow[t]{2}{*}{ Primer sequence $\left(5^{\prime} \rightarrow 3^{\prime}\right)$} & \multirow{2}{*}{ Motif } & \multirow{2}{*}{$\begin{array}{l}\mathrm{Tm} \\
\left({ }^{\circ} \mathrm{C}\right)\end{array}$} & \multirow{2}{*}{$\begin{array}{l}\text { PCR } \\
\text { fragment } \\
\text { (bp) }\end{array}$} & \multirow{2}{*}{ PIC } & \multirow{2}{*}{ Putative function } \\
\hline & & cDNA & SSR & & & & & & \\
\hline \multirow[t]{2}{*}{1} & \multirow[t]{2}{*}{ PBM031 } & \multirow[t]{2}{*}{ FP094740 } & \multirow[t]{2}{*}{ GU644371 } & CGCCGAGTTCCCTATTATTATTI & \multirow[t]{2}{*}{$(A G)_{6}-(A G)_{7}$} & \multirow[t]{2}{*}{56} & \multirow[t]{2}{*}{191} & \multirow[t]{2}{*}{0} & \multirow[t]{2}{*}{ MYB-like transcription factor } \\
\hline & & & & AGCACAGCCTCCGTGATTG & & & & & \\
\hline \multirow[t]{2}{*}{2} & \multirow[t]{2}{*}{ PBM032 } & \multirow[t]{2}{*}{ FP098085 } & \multirow[t]{2}{*}{ GU644372 } & ПТТCCCAAATAAAACCTCACC & \multirow[t]{2}{*}{$(\mathrm{CCG})_{7}-(\mathrm{CCT})_{6}$} & 56 & 143 & 0 & PHD finger protein \\
\hline & & & & GTCCATTAGGGTTCCACTGA & & & & & \\
\hline 3 & PBM033 & FP099510 & GU644373 & CTGACTGTGCGTGCGTCTC & $(\mathrm{CG})_{8}(\mathrm{AG})_{14}$ & 56 & 155 & 0 & Small GTP-binding protein \\
\hline & & & & CTTGGTCTCGCTCATCTCCTC & & & & & \\
\hline 4 & PBM034 & FP098748 & GU644374 & TCGGCTCGGCGTGATGGAT & $(\mathrm{GAG})_{5}(\mathrm{GCG})_{5}$ & 62 & 169 & 0 & GTP binding protein \\
\hline & & & & ATCGGCATCCGCGACTGCC & & & & & \\
\hline 5 & PBM035 & FP100911 & GU644375 & ACCGTGATGACTACCGCCGCGACC & $(\mathrm{GTG})_{7}-(\mathrm{GTG})_{7}$ & 62 & 165 & 0.368 & U2 snRNP auxiliary factor \\
\hline & & & & TGCTGCCTCCACCCCTCCGTCC & & & & & \\
\hline 6 & PBM036 & FP096684 & GU644376 & CACATGGACCGCCTCATCC & $(T A)_{8}$ & 47 & 169 & 0.259 & Polypeptide-associated complex \\
\hline & & & & GCAACAAAACGAGAACCAGAC & & & & & \\
\hline 7 & PBM037 & FP101192 & GU644377 & TGCAAGCCTGCTATACGTT & $(T A)_{7}-(T A)_{6}$ & 47 & 130 & 0 & Thaumatin family protein \\
\hline & & & & GAAGTGGGAGTACATACTTCCCA & & & & & \\
\hline 8 & PBM038 & FP101125 & GU644378 & GGTCGGCTCATITGTAGTGT & $(\mathrm{TC})_{9}(\mathrm{TA})_{22}$ & 48 & 210 & 0.365 & GCIP-interacting \\
\hline & & & & CAACCTTCAGGCAATAGATTACAT & & & & & \\
\hline 9 & PBM039 & FP091409 & GU644379 & CATCCTCAGTITCTCACCG & $(\mathrm{TC})_{12}-(\mathrm{CTT})_{6}$ & 53 & 171 & 0.355 & Unknown protein \\
\hline & & & & CAGCTTCACCAACTTGTGG & & & & & \\
\hline 10 & PBM040 & FP096343 & GU644380 & GAATCATCTGGGAAGAAGAAGGA & $(\mathrm{TC})_{7}-(\mathrm{TC})_{7}$ & 51 & 178 & 0 & Bicolor hypothetical protein \\
\hline & & & & TGCATTGCATTTGGCTTAGTAGT & & & & & \\
\hline 11 & PBM041 & FP095242 & GU644381 & TGGTGTTGCCTGTGACCTTAC & $(\mathrm{TG})_{8}(\mathrm{AG})_{10}$ & 53 & 167 & 0 & typeA response regulator 1 \\
\hline & & & & СCCACCTCCACCTCTACTACG & & & & & \\
\hline 12 & PBM042 & FP093940 & GU644382 & TCCTITACGGCTITACCCC & $(G A)_{7}-(A G)_{6}$ & 53 & 156 & 0.365 & SAM and SH3 domain- \\
\hline & & & & GCCCCAGCTTAGTACACCAC & & & & & \\
\hline 13 & PBM043 & FP099127 & GU644383 & CTCACCGCCCCACCTCGCA & $(A G)_{13}$ & 60 & 128 & 0 & IAA15 - auxin-responsive \\
\hline & & & & CGGCTGCTGATGCGGAGGA & & & & & \\
\hline 14 & PBM044 & FP095585 & GU644384 & AAGGCCCACGTTGCCAGAC & $(A G)_{20}$ & 55 & 173 & 0.371 & Bicolor hypothetical protein \\
\hline & & & & GTTCCCGTTGATGCCCCAC & & & & & \\
\hline 15 & PBM045 & FP098751 & GU644385 & TGAGCGAGGTAGTICATITAGTAA & $(C A)_{20}$ & 53 & 132 & 0.322 & DRE binding factor \\
\hline & & & & CCTACGACGAGTAGATTGCGAGT & & & & & \\
\hline 16 & PBM046 & FP094276 & GU644386 & CTCAGAGCAGACACTGCTTATTCC & $(\mathrm{CT})_{5}-(\mathrm{CT})_{6}$ & 50 & 102 & 0.395 & Unknown protein \\
\hline & & & & GCGTCTTCATTGCAGCCATCT & & & & & \\
\hline 17 & PBM047 & FP099829 & GU644387 & ACCACGTTGCAGGATTCACT & $(\mathrm{CT})_{13}$ & 53 & 119 & 0 & Bicolor hypothetical protein \\
\hline & & & & CGATGAGCAGCACAACAGC & & & & & \\
\hline 18 & PBM048 & FP092637 & GU644388 & GCAAAAGAGCGCACTTGAC & $(\mathrm{CT})_{27}$ & 53 & 163 & 0 & Serine carboxypeptidase \\
\hline & & & & GGAGGACACTAGAGTTGGCATT & & & & & \\
\hline 19 & PBM049 & FP099913 & GU644389 & ACAGCAGATAGTCCCAAAAT & $(G A)_{14}$ & 50 & 117 & 0.305 & Unknown protein \\
\hline & & & & GACAGCAGGATGAAGAGCA & & & & & \\
\hline 20 & PBM050 & FP093015 & GU644390 & AGTATAGTATGTTCGTITAAGTGG & $(C A)_{11}$ & 45 & 137 & 0 & Oxidoreductase \\
\hline & & & & TGTAATGTTIAAGGTTCCGT & & & & & \\
\hline 21 & PBM051 & FP092618 & GU644391 & AGACATTGTCAACTGTAAGTTGGTAGAG & $(\mathrm{TC})_{23}$ & 50 & 111 & 0 & VQ motif family protein \\
\hline
\end{tabular}
(FP099842) TITACAAGCAATACACCCAGAAATAG 
Table 3 Characteristics of the $\boldsymbol{P}$. pubescens SSR markers derived from FL-cDNAs (Continued)

\begin{tabular}{|c|c|c|c|c|c|c|c|c|c|}
\hline \multirow[t]{2}{*}{22} & PBM052 & FP095787 & GU644392 & AGCGGGCAGGCTATGTATT & $(\mathrm{TCT})_{11}$ & 51 & 140 & 0.359 & ELF4-like protein \\
\hline & & & & TTGCTTCTCCCCTAATGACA & & & & & \\
\hline \multirow[t]{2}{*}{23} & PBM053 & FP094717 & GU644393 & CCCCATAATCTGCTCCCTTCT & $(T \mathrm{C})_{10}$ & 51 & 102 & 0 & $\begin{array}{l}\text { KN1-type homeobox } \\
\text { transcription factor }\end{array}$ \\
\hline & & & & GGTTCTTGGCGTATGGTATGTTC & & & & & \\
\hline \multirow[t]{2}{*}{24} & PBM054 & FP100158 & GU644394 & ATCGGGAGGGATGCGGCAGC & $(\mathrm{GGCGGA})_{6}$ & 62 & 121 & 0.305 & Unknown protein \\
\hline & & & & GCGGACCAAGCGGAACACC & & & & & \\
\hline \multirow[t]{2}{*}{25} & PBM055 & FP100601 & GU644395 & CATGGATGTTGTTGAGTGAGGC & $(\mathrm{TC})_{7}$ & 53 & 199 & 0 & Nonspecific lipid-transfer \\
\hline & & & & GCACAAAGACTAGTACTCGAGGTGG & & & & & \\
\hline \multirow[t]{2}{*}{26} & PBM056 & FP100601 & GU644396 & CATGGATGTTGTTGAGTTGAGGC & $(\text { CTCCAT })_{6}$ & 53 & 177 & 0 & Nonspecific lipid-transfer \\
\hline & & & & GCACAAAGACTAGTACTCGAGGTGG & & & & & \\
\hline \multirow[t]{2}{*}{27} & PBM057 & FP097951 & GU644397 & CGCCCACCCCTCCTTCGTCT & $(\mathrm{ACACAG})_{5}$ & 59 & 111 & 0 & Cp protein \\
\hline & & & & TCCTTGGCACGGCCACTCA & & & & & \\
\hline \multirow[t]{2}{*}{28} & PBM058 & FP097794 & GU644398 & GGCCGAGATCCTCCTTTCT & $(\mathrm{GGCGGT})_{5}$ & 59 & 171 & 0 & Unknown protein \\
\hline & & & & CCATCCCCGCCTTCACCAC & & & & & \\
\hline \multirow[t]{2}{*}{29} & PBM059 & FP094127 & GU644399 & ATTAGTCACGCACCGAGAAGGAA & $(\mathrm{AGATG})_{6}$ & 55 & 172 & 0 & Transcription elongation \\
\hline & & & & AGACGCAAGAACTCGACAGGGA & & & & & \\
\hline \multirow[t]{2}{*}{30} & PBM060 & FP101691 & GU644400 & CACGCCAGCTCCAGATGCCACCAT & $(\mathrm{CACCC})_{5}$ & 59 & 119 & 0 & Sucrose transporter \\
\hline & & & & TGCCCTTCCACCTCCTCTGACCTCC & & & & & \\
\hline \multirow[t]{2}{*}{31} & PBM061 & FP095238 & GU644401 & СССТАТСССАТССТССТССС & $(\mathrm{CCTCT})_{5}$ & 55 & 119 & 0 & Smr domain containing \\
\hline & & & & GGTTGCTCACTITCCTGCTCC & & & & & \\
\hline \multirow[t]{2}{*}{32} & PBM062 & FP096136 & GU644402 & TGCTGGTTGGGTTCATCACGA & $(\mathrm{TTCT})_{7}$ & 53 & 156 & 0 & Bicolor hypothetical protein \\
\hline & & & & GAGGGTTACAACAGGGGCAAAGA & & & & & \\
\hline \multirow[t]{2}{*}{33} & PBM063 & FP098746 & GU644403 & CAACGCAACGCCATTCCAAACA & $(\mathrm{TCCA})_{5}$ & 59 & 138 & 0 & U-box domain \\
\hline & & & & CACCTCCAGGCCCTGGTACTCCA & & & & & \\
\hline \multirow[t]{2}{*}{34} & PBM064 & FP099572 & GU644404 & CATTTCTCATTGCCGCTGTAAC & $(\mathrm{GAGT})_{5}$ & 53 & 139 & 0 & Unknown protein \\
\hline & & & & TCCTITGCCCTCCTCTTCCT & & & & & \\
\hline \multirow[t]{2}{*}{35} & PBM065 & FP096965 & GU644405 & GTCAGTCAGGCGGCACGAG & $(\mathrm{CG})_{5}-(\mathrm{CGG})_{9}$ & 60 & 183 & 0 & Bicolor hypothetical protein \\
\hline & & & & CGCGTAGGACGAGATCACCTC & & & & & \\
\hline \multirow[t]{2}{*}{36} & PBM066 & FP095562 & GU644406 & СTCTTCACCGAAACCGAAAG & $(\mathrm{CGG})_{9}$ & 57 & 137 & 0.477 & Spliceosomal protein \\
\hline & & & & CGTTGAGGTTCCTGAGGTAGAC & & & & & \\
\hline \multirow[t]{2}{*}{37} & PBM067 & FP098504 & GU644407 & GGTGCGGGTGCAGTTATT & $(\mathrm{CTT})_{8}$ & 51 & 185 & 0 & RNA-binding protein \\
\hline & & & & AGCATCATCCGCCAGAATA & & & & & \\
\hline \multirow[t]{2}{*}{38} & PBM068 & FP093884 & GU644408 & AACCGTGCACTACTTGCTCT & $(\mathrm{TCT})_{8}$ & 51 & 155 & 0 & pollenless3 mRNA \\
\hline & & & & ACCTTGTGGACGACATGGA & & & & & \\
\hline \multirow[t]{2}{*}{39} & PBM069 & FP099427 & GU644409 & СCСТTССССTCAACAACAA & $(\mathrm{CCG})_{8}$ & 57 & 101 & 0.360 & Alba superfamily protein \\
\hline & & & & TACCGATCCATGGCTCCTT & & & & & \\
\hline \multirow[t]{2}{*}{40} & PBM070 & FP094239 & GU644410 & TCGTGCCTITCGCCTCCTG & $(\mathrm{TCT})_{7}$ & 55 & 117 & 0 & Bicolor hypothetical protein \\
\hline & & & & CTGTACGGCCCGAACTTGTA & & & & & \\
\hline \multirow[t]{2}{*}{41} & PBM071 & FP093953 & GU644411 & AGCGTCACCTCCGCCTTCT & $(\mathrm{CGA})_{8}$ & 57 & 101 & 0 & Unknown protein \\
\hline & & & & TCCTTGGCCTCGTCTTGGT & & & & & \\
\hline \multirow[t]{2}{*}{42} & PBM072 & FP093285 & GU644412 & CCTCCCACTGTCACGGCACC & $(\mathrm{CTC})_{9}$ & 59 & 116 & 0 & Bicolor hypothetical protein \\
\hline & & & & GGCTGTGGCGACAAGGCTG & & & & & \\
\hline \multirow[t]{2}{*}{43} & PBM073 & FP096973 & GU644413 & AGCAGCTCTACGGCAAGAAGAAG & $(\text { ATC })_{8}$ & 53 & 139 & 0 & Bicolor hypothetical protein \\
\hline & & & & TGCAGCCTTGAGGAATTGAGAA & & & & & \\
\hline
\end{tabular}


Table 3 Characteristics of the $\boldsymbol{P}$. pubescens SSR markers derived from FL-cDNAs (Continued)

\begin{tabular}{|c|c|c|c|c|c|c|c|c|c|}
\hline \multirow[t]{2}{*}{44} & \multirow[t]{2}{*}{ PBM074 } & \multirow[t]{2}{*}{ FP096816 } & \multirow[t]{2}{*}{ GU644414 } & CCCACCGAAGTAATCACGC & \multirow[t]{2}{*}{$(\mathrm{CTC})_{8}$} & \multirow[t]{2}{*}{55} & \multirow[t]{2}{*}{119} & \multirow[t]{2}{*}{0} & \multirow{2}{*}{$\begin{array}{l}\text { Transcription factor } \\
\text { HBP-1a(c14) }\end{array}$} \\
\hline & & & & CTCGCACAACAAAAGAAATCA & & & & & \\
\hline \multirow[t]{2}{*}{45} & \multirow[t]{2}{*}{ PBM075 } & \multirow[t]{2}{*}{ FP096707 } & \multirow[t]{2}{*}{ GU644415 } & AGTTCCTTCTTCCTTCCTTCCGTGGTG & \multirow[t]{2}{*}{$(G A A)_{8}$} & \multirow[t]{2}{*}{53} & \multirow[t]{2}{*}{101} & \multirow[t]{2}{*}{0.510} & \multirow[t]{2}{*}{ Unknown protein } \\
\hline & & & & CGGCATTGCGATTGTGC & & & & & \\
\hline \multirow[t]{2}{*}{46} & \multirow[t]{2}{*}{ PBM076 } & \multirow[t]{2}{*}{ FP101632 } & \multirow[t]{2}{*}{ GU644416 } & ATGCCTTCACCACACTTAC & $(\mathrm{GCA})_{8}$ & 51 & 121 & 0 & AP2/ERF domain protein \\
\hline & & & & CATCGTGATGTCTCCAATC & & & & & \\
\hline 47 & PBM077 & FP096443 & GU644417 & CCGCTTCCTCCCACCAAAT & $(\mathrm{CCG})_{5}$ & 59 & 181 & 0 & Bicolor hypothetical protein \\
\hline & & & & CGCAGTACAGCAGCTCCCC & & & & & \\
\hline 48 & PBM078 & FP095554 & GU644418 & CCCAAATCCAACCAGAACCA & $(\mathrm{CGG})_{11}$ & 59 & 187 & 0 & Anti-silencing protein \\
\hline & & & & GGAGGAGGCATTCGTAGGAGA & & & & & \\
\hline 49 & PBM079 & FP097911 & GU644419 & AAGGATGGTAACGTACATACA & $(\mathrm{AT})_{5}$ & 44 & 159 & 0 & Unknown protein \\
\hline & & (FP092888) & & CATGACAAATTAAAGGTATCA & & & & & \\
\hline 50 & PBM080 & FP093425 & GU644420 & CGAGGTTCTTGGGCTCAGTT & $(A G)_{13}$ & 53 & 116 & 0.375 & ATP binding protein \\
\hline & & & & ACACGCCTCCAATAAAACAAAC & & & & & \\
\hline 51 & PBM081 & FP097485 & GU644421 & TCTACTCCGTAGCCGCCTTC & $(\mathrm{CT})_{16}$ & 56 & 135 & 0 & Pyridoxamine 5-phosphate \\
\hline & & & & AGAGCCTCCATTGGATGGG & & & & & \\
\hline 52 & PBM082 & FP099753 & GU644422 & AATTGTTGCCCTGCCTAGCT & $(\mathrm{TC})_{5}-(\mathrm{TC})_{16}$ & 53 & 148 & 0 & Homeodomain leucine-zipper \\
\hline & & & & GCAAGATGAGAAGAATTAAAGCTGC & & & & & protei \\
\hline 53 & PBM083 & FP101428 & GU644423 & CCATTTGGCATTTGCTCCC & $(G A)_{15}$ & 59 & 186 & 0 & GTPase SAR1 (Sar1.1) \\
\hline & & & & GCACCCCGTAGAACCAGTCC & & & & & \\
\hline 54 & PBM084 & FP092513 & GU644424 & CTTCTCATGGGGTCAGCTACTC & $(\mathrm{TC})_{17}(\mathrm{AC})_{16}$ & 53 & 201 & 0.369 & Brown planthopper-induced \\
\hline & & & & ATCACTTCTGCGATCTTGGTC & & & & & resist \\
\hline 55 & PBM085 & FP091409 & GU644425 & GGGGAGCCATCCTCAGTT & $(\mathrm{TC})_{12}-(\mathrm{CTT})_{6}$ & 55 & 183 & 0.346 & Putative precursor micro \\
\hline & & & & GCTGGCAGCTTCACCAACT & & & & & \\
\hline 56 & PBM086 & FP096167 & GU644426 & GTGGAAAATAAAGAAGCGC & $(\mathrm{TC})_{9}-(\mathrm{TC})_{9}$ & 51 & 139 & 0 & Unknown protein \\
\hline & & & & TTCCTGCTITGATCTTGC & & & & & \\
\hline 57 & PBM087 & FP093957 & GU644427 & ACCCCAAGCATCCCCAAAA & $(\mathrm{CCT})_{5}-(\mathrm{CGC})_{9}$ & 59 & 166 & 0.373 & Bicolor hypothetical protein \\
\hline & & & & CCGCAGGGAAGTCGAAGGTC & & & & & \\
\hline 58 & PBM088 & FP091571 & GU644428 & GTGTATTGGCTTTCCAGCTITTCC & $(A G)_{11}$ & 55 & 211 & 0 & $\begin{array}{l}\text { Knotted class } 1 \\
\text { homeodomain protein }\end{array}$ \\
\hline & & & & TCTCCGCACGCTACTGTCCC & & & & & \\
\hline 59 & PBM089 & FP097920 & GU644429 & TCCCTTATCCACCAAACACGC & $(\mathrm{CT})_{17}$ & 56 & 172 & 0.369 & Bicolor hypothetical protein \\
\hline & & & & GCTGGCAACGACGCACCTC & & & & & \\
\hline 60 & PBM090 & FP097267 & GU644430 & AGAGTCGGATAAGGGTAGCG & $(A G)_{12}$ & 53 & 106 & 0.195 & Repair protein RAD23 \\
\hline & & & & CGATCTCGAAGTTCGTGCC & & & & & \\
\hline 61 & PBM091 & FP100553 & GU644431 & ATAGAGGCATACAGCCGCAGAC & $(A G)_{14}$ & 56 & 126 & 0.369 & Macrophage migration \\
\hline & & & & TAGGCACGGCATCACGGAC & & & & & \\
\hline 62 & PBM092 & FP099642 & GU644432 & GAACGCCGCATCCAGCCTCT & $(\mathrm{TC})_{13}$ & 53 & 155 & 0 & Basic/leucine zipper protein \\
\hline & & & & GGTCGGGTCCTTGGACAAAC & & & & & \\
\hline 63 & PBM093 & FP100738 & GU644433 & TCGCAGTAAACAGTCTCATCACATC & $(\mathrm{CCT})_{8}$ & 59 & 150 & 0 & Disulfide isomerase (PDIL2-2) \\
\hline & & & & TCAGGGCCACCACCTCGTCT & & & & & \\
\hline 64 & PBM094 & FP095169 & GU644434 & GATTGAGGAGCCCCAAACC & $(C C G)_{8}$ & 57 & 257 & 0 & DUF2372 superfamily protein \\
\hline & & & & CACAACAACCGCAAGAGCC & & & & & \\
\hline 65 & PBM095 & FP098630 & GU644435 & TATTAGTCGAGTTTGGGTCTCC & $(\mathrm{CCT})_{8}$ & 55 & 115 & 0.430 & Unknown protein \\
\hline & & & & GGTGAACGGCATGGCTGCT & & & & & \\
\hline
\end{tabular}


Table 3 Characteristics of the $\boldsymbol{P}$. pubescens SSR markers derived from FL-cDNAs (Continued)

\begin{tabular}{|c|c|c|c|c|c|c|c|c|c|}
\hline \multirow[t]{2}{*}{66} & PBM096 & FP100124 & GU644436 & CACTCGGCTCGTCCTCGTCT & $(\mathrm{CCTC})_{6}$ & 60 & 129 & 0 & PLAC8 superfamily protein \\
\hline & & & & AGGGTGGCTAAGGCTCGTCTC & & & & & \\
\hline \multirow[t]{2}{*}{67} & PBM097 & FP099849 & GU644437 & CTGCCACTCCATCCCTGCC & $(\mathrm{CACGCG})_{5}$ & 59 & 101 & 0 & Unknown protein \\
\hline & & & & CTCGATGGCGACGGCTGTT & & & & & \\
\hline \multirow[t]{2}{*}{68} & PBM098 & FP097471 & GU644438 & CCCCGTCTTCTCGTCGTCT & $(\mathrm{TCGCCG})_{5}$ & 56 & 169 & 0 & BAH_BAHCC1 superfamily \\
\hline & & & & GACTITGTCGGAGCCCTTGA & & & & & \\
\hline
\end{tabular}

species with a large genome (data unpublished) in which we developed 15 EST-SSR markers from 52 promising sequences selected from 3406 non-redundant ESTs (Dong et al. 2011).

We surveyed the allelic variability of the markers by genotyping 50 open-pollinated seedlings germinated from the year 2010 seedlot (Table 3). Among the 68 FLcDNA SSR markers, only 22 (32.4\%) showed polymorphism. The polymorphism information content (PIC) values of the 68 markers ranged from 0 to 0.51 with a mean value of 0.12 . For the 22 polymorphic loci, the PIC values ranged from 0.19 to 0.51 with a mean value of 0.36 , and the top ten markers in terms of polymorphism were PBM075, PBM069, PBM095, PBM046, PBM066, PBM080, PBm087, PBM044, PBM084 and PBM091. SSR polymorphism in $P$. pubescens is much lower than observed in cereals (Thiel et al. 2003; Yu et al. 2004), coffee (Aggarwal et al. 2007) and the rubber tree (Feng et al. 2009). Bamboo P. pubescens has a long flowering interval of more than 60 years (Janzen 1976; Watanabe et al. 1982). Therefore, open pollination (DNA recombination) appears to have limited the amount of replication slippage, which diversifies SSR alleles (Richards and Sutherland 1994; Jakupiak and Wells 1999). Clonal propagation in the interim periods of flowering has reduced the SSR diversity in bamboo (Nayak and Rout 2005). In a previous study, we discovered almost no allelic variation in the panel of 11 varieties and 17 provenances of $P$. pubescens using 19 GSS-SSRs (Tang et al. 2010).

Interspecific transferability and polymorphism of $P$. pubescens FL-cDNA SSR markers

Although more than 1000 bamboo species have been described, the vast majority of publically-available sequence data are derived from P. pubescens (Tang 2009). Therefore, the development of a set of transferable $P$. pubescens FLcDNA SSR markers suitable for other bamboo species would help to accelerate genetic research and comparative genomics in the Bambusoideae subfamily. Previously, we developed $19 P$. pubescens GSS-SSR markers and successfully transferred them to six other Phyllostachys species with an average transferability of $75.3 \%$ and $66.7 \%$ polymorphism (Tang et al. 2010). In B. arundinacea, 100\% and $83.3 \%$ transferability were achieved with 6 SSR markers in eight other Bambusa species and 10 species of other genera, respectively (Nayak and Rout 2005). In B. oldhamii, we achieved an average 59.6\% transferability and $51.4 \%$ polymorphism with 15 markers in 14 bamboo species including four species within the same genus (Dong et al. 2011). We tested the transferability and polymorphism of these 68 putative FL-cDNA SSR markers across 41 diverse species in six tribes of the Bambusoideae subfamily, as defined by Das et al. (2008) and Yang et al. (2008) (Additional file 2: Table S1 and Additional file 3: Table S2). Successful amplification became less likely with increasing phylogenetic distance from $P$. pubescens, with an $83.1 \%$ success rate within the genus Phyllostachys, a $79.4 \%$ success rate across genera within the subtribe Shibataeeae, and a $49.3 \%$ average success rate for other subtribes, ranging from 36.8-76.5\% (Table 4 and Figure 1). In contrast, the number of markers showing polymorphism increased with phylogenetic distance, with $79.4 \%$ of markers showing polymorphism within the genus Phyllostachys, 91.3\% showing polymorphism within the Shibataeeae, and 92.8\% showing polymorphism when comparing other subtribes. Markers in coding sequences were on average the most transferrable $(69.1 \%)$ and the least polymorphic (89.4\%), compared to those located in $5^{\prime}$-UTRs $(63.4 \%$

Table 4 Transferability/polymorphism of $P$. pubescens FL-cDNA-derived SSR markers across species and genera in the Bambusoideae subfamily

\begin{tabular}{|c|c|c|c|c|c|c|c|c|}
\hline \multirow{2}{*}{$\begin{array}{l}\text { Types of } \\
\text { EST-SSR } \\
\text { (number) }\end{array}$} & \multirow{2}{*}{$\begin{array}{l}\text { Intra-genus } \\
\text { (Phyllostachys) }\end{array}$} & \multirow{2}{*}{$\begin{array}{l}\text { Inter-genus within } \\
\text { substribe (Shibataeeae) }\end{array}$} & \multicolumn{5}{|l|}{ Inter-substribe } & \multirow[t]{2}{*}{ Average } \\
\hline & & & Melocanninae & Bambusinae & Chusqueeae & Arundinarieae & Guaduinae & \\
\hline 5'-UTR (41) & $85.8 \% / 81.4 \%$ & $69.2 \% / 91.3 \%$ & $42.7 \% / 93.6 \%$ & $35.5 \% / 94.4 \%$ & $41.5 \% / 88.2 \%$ & $78.1 \% / 91.4 \%$ & $29.3 \% / 90.9 \%$ & $63.4 \% / 90.7 \%$ \\
\hline ORF (18) & $80.6 \% / 71.3 \%$ & $70.1 \% / 91.0 \%$ & $58.3 \% / 97.7 \%$ & $57.1 \% / 95.7 \%$ & $61.1 \% / 90.9 \%$ & $75.6 \% / 89.1 \%$ & $55.6 \% / 95.8 \%$ & $69.1 \% / 89.4 \%$ \\
\hline $3^{\prime}-U T R(9)$ & $75.9 \% / 86.6 \%$ & $72.2 \% / 91.7 \%$ & $41.7 \% / 95.0 \%$ & $46.0 \% / 96.4 \%$ & $22.2 \% / 100.0 \%$ & $70.9 \% / 86.9 \%$ & $33.3 \% / 100.0 \%$ & $61.8 \% / 91.4 \%$ \\
\hline Average & $83.1 \% / 79.4 \%$ & $69.9 \% / 91.3 \%$ & $46.7 \% / 94.9 \%$ & $42.6 \% / 95.0 \%$ & $44.1 \% / 90.5 \%$ & $76.5 \% / 90.2 \%$ & $36.8 \% / 93.4 \%$ & \\
\hline
\end{tabular}




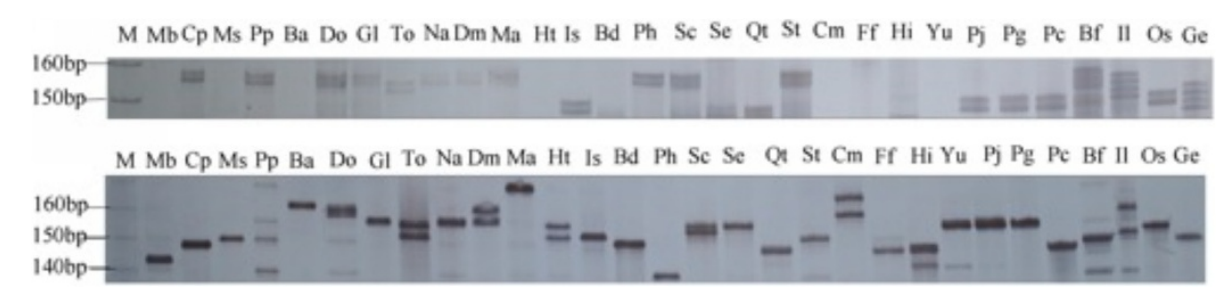

Figure 1 Polyacrylamide gel electrophoresis bands representing microsatellites derived from FL-cDNA sequences, tested on a panel of selected bamboo species to evaluate transferability and polymorphism in locus of PBM042 (above) and PBM064 (nether). M: size marker. Mb, Cp, etc.: Bamboo species abbreviations are listed in Supplementary Table 1.

transferrable, $90.7 \%$ polymorphic) and 3 '-UTRs (61.8\% transferrable, $91.4 \%$ polymorphic). These trends were exacerbated with increasing phylogenetic distance. These matches the results from a metastudy of 601 loci in 35 plant species showing an average $89.8 \%$ transferability at the subgenus level, $76.4 \%$ at the genus level and $35.2 \%$ at the family level (Rossetto 2001). Interestingly, more than $17(25 \%)$ of the markers were transferrable to more than $85 \%$ of the tested species (Additional file 3: Table S2). This success rate suggests that FL-cDNA SSRs and their flanking regions are sufficiently conserved (Zhang et al. 2005), and it is therefore possible to transfer P. pubescens FL-cDNA SSR markers to other bamboo species for evolutionary studies and phylogenetic reconstructions (Sharma et al. 2008).

\section{Using polymorphic FL-cDNA SSR markers to estimate outcrossing rates and identify interspecific bamboo hybrids}

Sexual propagation increases genetic diversity by creating progenies of different genotypes through recombination (i.e. outcrossing). This is advantageous for predominantly clonal plants such as most bamboo species, which rely mostly on vegetative regeneration interspersed with occasional flowering (Janzen 1976). The analysis of the reproductive system is therefore fundamental to elucidate primary genetic diversity and the structure of regenerating bamboo populations, and to adopt strategies for genetic improvement. Previous studies on the bamboo reproductive system based on field data and artificial pollination showed that self-compatibility is predominant in Sasa species (Nishiwaki and Konno 1990), and the selfing rate could approach 0.99 in Merostachys riedeliana (Guilherme and Ressel 2001). Outcrossing rate was estimated using SSR-based analysis as reported in S. cernua (Kitamura and Kawahara 2011).

Among the 22 polymorphic SSR markers described above, the ten most polymorphic ( $\mathrm{PIC} \geq 0.36$ ) were used to detect polymorphisms in 50 open-pollinated half-sib seeds (year 2011) from three flowering sites in the Guangxi Province separated by at least $100 \mathrm{~km}$. Polymorphism in the PBM044, PBM080 and PBM095 loci was identical in the seeds from all three flowering sites, whereas PBM084 and PBM091 featured additional alleles from Lipu, PBM069, PBM075, PBM087 and PBM091 featured additional alleles from Lingchuan, and PBM069, PBM075 and PBM084 featured additional alleles from Guanyang (Table 5). This indicated that flowering culms in different sites featured diverse SSR genotypes and produced genetically-diverse half-sib seed sources. Therefore, we used these eight polymorphic loci to estimate the outcrossing rates and other related genetic parameters for $P$. pubescens (Table 5). The overall estimates of $t m$ and $t s$ for three culms were 0.089 for both parameters, with no standard deviation. The estimates for individual culms showed small differences of 0.067 in Lipu and Lingchuan, and 0.133 in Guanyan, again for both parameters. Estimation of $F_{i s}$ for the overall population was 0.195, indicating homozygote excess. We found that the outcrossing rate was 0.089 , estimated from eight polymorphic multilocus

Table 5 Seed number, estimated outcrossing rates and relative parameters for each of three $P$. pubescens flowering culms at 8 loci

\begin{tabular}{|c|c|c|c|c|c|c|c|c|c|c|c|c|}
\hline \multirow{2}{*}{$\begin{array}{l}\text { Flowering site } \\
\text { (county) }\end{array}$} & \multirow[t]{2}{*}{$\mathbf{N}$} & \multirow[t]{2}{*}{$t m$} & \multirow[t]{2}{*}{ ts } & \multirow[t]{2}{*}{ Fis } & \multicolumn{8}{|c|}{ Genotype } \\
\hline & & & & & PBM044 & PBM069 & PBM075 & PBM080 & PBM084 & PBM087 & PBM091 & PBM095 \\
\hline Lipu & 50 & $0.067(0.0)$ & $0.066(0.0)$ & 0.182 & $p$ & & & $p$ & $p$ & & $p$ & $p$ \\
\hline Lingchuan & 50 & $0.067(0.0)$ & $0.067(0.0)$ & 0.173 & $p$ & $p$ & $p$ & $p$ & & $p$ & $p$ & $p$ \\
\hline Guanyan & 50 & $0.133(0.0)$ & $0.135(0.0)$ & 0.231 & $p$ & $p$ & $\mathrm{p}$ & $p$ & $\mathrm{p}$ & & & $p$ \\
\hline Average & & $0.089(0.0)$ & $0.089(0.0)$ & 0.195 & & & & & & & & \\
\hline
\end{tabular}

$\mathrm{N}$ the number of analyzed seeds; tm multi-locus outcrossing rate and standard error in parentheses; $t$, single-locus outcrossing rate and standard error in parentheses. Fis inbreeding coefficient; p polymorphism. 
datasets in $P$. pubescens, which is slight lower than the 0.148 reported in S. cernua using six multilocus SSR datasets (Kitamura and Kawahara 2011). This indicated that the reproductive system of $P$. pubescens predominantly involves self-fertilization with an adequate proportion of crossing to ensure genetic diversity as reported for S. cernua (Kitamura and Kawahara 2011).

The grow-out test for bamboo interspecific hybrids is time-consuming and laborious because it involves growing plants to maturity (which takes at least 5 years), assessing several anatomical, morphological and floral (long-term interval) characteristics that distinguish the hybrid. The polymorphic SSR markers could also help in the rapid and accurate identification of interspecies hybrids, as reported in poplar (Rajora and Rahman 2003) and wheat-barley (Malysheva et al. 2003). To obtain proof of principle that our novel SSR markers are suitable for hybrid characterization, we next selected several highlytransferable and polymorphic FL-cDNA SSR markers. PBM032, PMB049, PMB063 and PMB064, each with a number of species-restricted alleles, were used to test uncharacterized bamboo samples. Marker PMB063 identified the parental species in one hybrid as $P$. kwangsiensis and $P$. bambusoides, because all sequenced bands contained the (TCCA $)_{n}$ motif although with a variable number of repeats (Figure 2). Similarly, marker PMB064 identified the parental species B. pervariabilis and Dendrocalamus latiflorus which are distantly related to $P$. pubescens, with a variable number of repeats in the $(\text { GAGT })_{n}$ motif (Figure 3). As previously shown using GSS-SSR markers, such high levels of transferability and polymorphism within the Bambusoideae subfamily should allow the use of FL-cDNA SSR markers to identify interspecific hybrids and their parents, both within the genus Phyllostachys (Tang et al. 2010) and in more distant taxa within subtribe of Shibataeeae (Lu et al. 2009). We have also developed several putative EST-SSR markers in B. oldhamii and have used these to identify some other sympodial bamboo interspecies hybrids (Wu et al. 2009; Dong et al. 2011). The SSR markers developed in the present study were used to identify not only interspecific hybrids from monopodial Phyllostachys but also intergeneric hybrids with sympodial rhizomes, which are distantly related to P. pubescens. Our data confirmed that microsatellites, especially SSR markers based on cDNAs and ESTs, are ideal for the identification of bamboo interspecies hybrids.

\section{Conclusions}

Our data provide insight into the association between SSRs and TEs in FL-cDNAs from the P. pubescens transcriptome, allowing us to develop and evaluate $68 \mathrm{FL}-$ cDNA SSR markers that can be used in P. pubescens and partially for many other bamboo species, to estimate the reproductive system of $P$. pubescens and identify several interspecific hybrids. These FL-cDNA SSR markers enrich the molecular marker resources currently available for bamboo. When a large set of polymorphic markers becomes available, we can use genome-wide association mapping in bamboo, in the absence of structured populations, to identify markers for traits of interest that can be used for marker-assisted selection in the Bambusoideae subfamily.

\section{Methods}

\section{Full-length CDNA mining and SSR/TE detection}

We obtained 10,608 FL-cDNA sequences from NCBI Entrez (http://www.ncbi.nlm.nih.gov/) on July 1, 2010. These cDNA sequences were assembled from five cDNA libraries constructed from breaking-out shoots, young $(40-\mathrm{cm})$ shoots and young leaves from plants, and shoots and roots from germinated seeds (Peng et al. 2010). We used EST Trimmer (http://pgrc.ipk-gatersleben.de/misa/ download/est_trimmer.pl) to remove $\operatorname{poly}(\mathrm{A} / \mathrm{T})$ runs from the $5^{\prime}$ and $3^{\prime}$ ends until there were no occurrences of $(\mathrm{T})_{5}$ or $(\mathrm{A})_{5}$ within a 50 -bp range. Redundant sequences were eliminated and overlapping sequences were spliced together using CAP3 (http://seq.cs.iastate.edu/cap3.html) (Huang and Madan 1999).

After pre-treatment, we used MISA (http://pgrc.ipkgatersleben.de/misa/misa.html) to screen for SSRs including mononucleotide repeats $\geq 10 \mathrm{bp}$ in length, dinucleotide
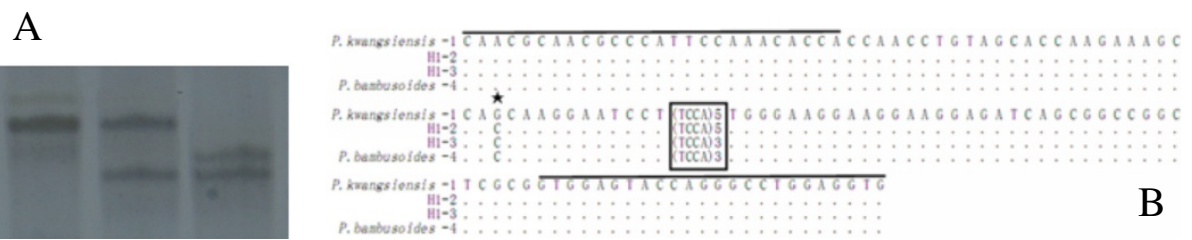

Figure 2 A, Microsatellite DNA fingerprints of $P$. kwangsiensis (line 1), P. bambusoides (line 3) and a presumed hybrid (line 2) at locus PBM063. B, Alignment of the nucleotide sequences of the microsatellite alleles at locus PBM014 amplified from P. kwangsiensis, P. bambusoides and two presumed hybrids. Nucleotides conserved among these sequences (relative to $P$. kwangsiensis) are shown by dots. The lines indicate the primer sequences used to amplify this microsatellite locus. The box highlights the microsatellite. The suffix numbers after bamboo species correspond to the DNA bands marked in part (a). 


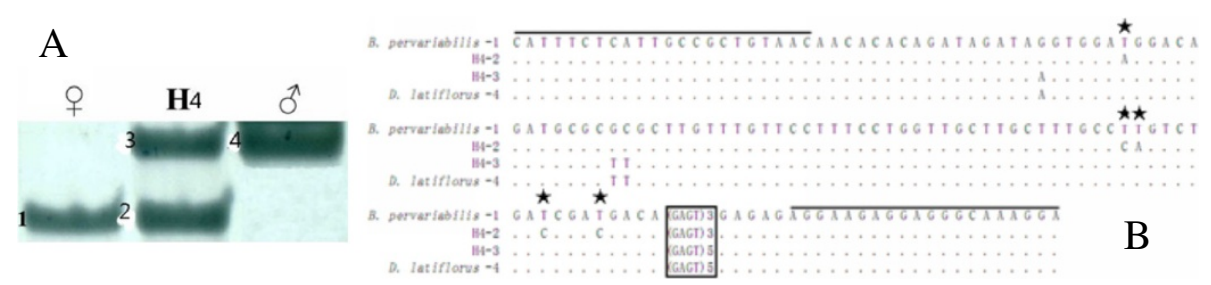

Figure 3 A, Microsatellite DNA fingerprints of Bambusa pervariabilis, Dendrocalamus latiflorus and their presumed hybrids at locus PBM064. B, Alignment of the nucleotide sequences of the microsatellite alleles at locus PBM064 amplified from B. pervariabilis, D. latiflorus and their presumed hybrids. Nucleotides conserved among these sequences (relative to B. pervariabilis) are shown by dots. The lines indicate the primer sequences used to amplify this microsatellite locus. The box highlights the microsatellite. The suffix numbers after the bamboo species correspond to the DNA bands marked in part (A).

to hexanucleotide repeats with $\geq 6$ repeat units, and interrupted composite SSRs with $\leq 100$ bp of intervening DNA. Putative annotations were assigned to non-redundant ESTs containing SSRs using BLAST against the Moso Bamboo cDNA Database (http://202.127.18.228/mbcd/) and the Gramene Ontologies Database (http://archive. gramene.org/plant_ontology/). TEs were identified using RepeatMasker and RepeatProteinMask (http://www. repeatmasker.org) based on similar elements present in the rice genome, and SSRs within TEs were screened using MISA with the same parameters as above. Additional file 1: Figure S1 provides a flow chart for the data mining and marker development process.

\section{Plant material and DNA extraction}

We used P. pubescens samples collected from the Anji Bamboo Germplasm Garden, Anji, Zhejiang Province, to identify and characterize putative FL-cDNA SSR markers. The polymorphism of these SSR markers was evaluated using 50 seedlings germinated from an open-pollinated seedlots (mixed seed sources, mainly from different flowering sites in the counties of Lipu, Lingchuan and Guanyang, Guangxi Province in the year 2010). Another 50 seedlings were germinated from open-pollinated half-sib seeds (year 2011) from three flowering culms in the same three counties ( $>100 \mathrm{~km}$ between sites) and were used to estimate the $P$. pubescens outcrossing rate. We obtained 41 representative bamboo species from 38 genera within six subtribes mainly found in China to test the transferability and polymorphism of the FL-cDNA SSR markers (Additional file 2: Table S1). We obtained three Phyllostachys interspecific hybrids from Jiangxi Province, China, and two intergeneric hybrids from Yoshinaka Bamboo Germplasm Garden, Fukuoka, Japan, for the hybrid identification tests. Genomic DNA was extracted from young leaves using the hexadecyltrimethylammonium bromide (CTAB) method (Doyle and Doyle 1987), with some modifications.

\section{Amplification and sequencing of SSR loci}

Primer pairs designed according to the available cDNA sequences were synthesized by Shanghai Sangon Biological
Engineering Technology \& Services Co., Ltd. P. pubescens DNA was amplified in 20- $\mu$ l reactions comprising 50 $100 \mathrm{ng}$ of template DNA, $0.2 \mu \mathrm{M}$ of each primer, $200 \mu \mathrm{M}$ of each dNTP and 1 unit of Taq DNA polymerase with $1 \times$ PCR universal buffer $\left(10 \mu \mathrm{M}\right.$ Tris- $\mathrm{HCl}, \mathrm{pH} 8.3$ at $25^{\circ} \mathrm{C}$; $50 \mu \mathrm{M} \mathrm{KCl}$ ), and $1.5 \mu \mathrm{M} \mathrm{MgCl}_{2}$ (Shanghai Sangon Biological Engineering Technology \& Services Co., Ltd). The reaction was heated to $95^{\circ} \mathrm{C}$ for 5 min using an ABI PE9700 thermocycler, followed by 30 cycles of $1 \mathrm{~min}$ denaturation at $95^{\circ} \mathrm{C}, 1 \mathrm{~min}$ annealing at $46-59^{\circ} \mathrm{C}$ depending on the primer pair (Table 3), and 2 min extension at $72^{\circ} \mathrm{C}$, followed by a final hold at $72^{\circ} \mathrm{C}$ for $5 \mathrm{~min}$. Amplified microsatellite loci were tested in 41 diverse species in six tribes of the Bambusoideae subfamily (Table 4) and interspecific hybrids (Figures 2 and 3). The annealing temperature was lowered by $2-5^{\circ} \mathrm{C}$ according to the evolutionary distance between species based on molecular markers (Das et al. 2008) and nuclear and chloroplast sequences (Yang et al. 2008), as suggested by Rossetto (2001). PCR products were separated on $6 \%$ polyacrylamide denaturing gels, and marker bands were revealed by silver staining as described by Panaud et al. (1996). Specific bands were excised directly from the silver staining polyacrylamide gel, purified using the EZ-10 Spin Column DNA Gel Extraction Kit (Biobasic Inc.) and ligated into the pUC18 vector (TaKaRa, Japan). Three positive clones for each bamboo species were selected for sequencing using BigDye terminator V3.1 in a cycle sequencing protocol according to the manufacturer's specifications (PE Applied Biosystems, ABI PRISM 3100-Avant Automatic DNA Sequencer). Vector sequences were removed then edited using Vector NTI software (version 10.0, Invitrogen Co., USA). Sequences were deposited in NCBI GenBank (accession nos GU644371-GU644438).

\section{Data analysis}

The polymorphism information content (PIC) (Botstein et al. 1980) of our SSR markers was determined using Powermarker v3.25 (Liu and Muse 2005). All 68 selected primer pairs were used to amplify template DNA from 
41 bamboo species covering 35 genera in six subtribes (Additional file 2: Table S1) and the statistical methods of Nayak and Rout (2005) and Sharma et al. (2009) were used to calculate the cross-taxon transferability and polymorphism (Additional file 3: Table S2), in which polymorphism is calculated only from the loci that were successfully transferred across taxa (Rossetto 2001). Single locus and multilocus outcrossing rates and relative parameters were analyzed separately under the mixed mating model of Ritland \& Jain (1981) and Ritland (2002), implemented using MLTR v3.4 (Ritland 1996).

\section{Additional files}

Additional file 1: Figure S1. Scheme used for database mining and the development of SSR markers from $P$. pubescens FL-CDNA sequences.

Additional file 2: Table S1. Species used to test cross species/genus amplification of $P$. pubescens FL-CDNA SSR loci.

Additional file 3: Table S2. Cross species/genus amplification of P. pubescens FL-cDNA SSR loci.

\section{Competing interests}

The authors declare that they have no competing interests.

\section{Authors' contribution}

DQT conceived the study, participated in its design, coordination, data analysis and interpretation, and drafted, reviewed and improved the manuscript. $Y L$ and JJL carried out genotyping, sequencing and the identification of hybrids. WJD and MBZ carried out mining of EST data, unigene prediction, and analysis of repeat type and frequency of microsatellites. WF and YI helped in interpretation the data and improve the manuscript. All authors have read and approved the final manuscript.

\section{Acknowledgements}

This work was financially supported by grants from the "973" Program (2012CB723008), National Natural Science Foundation of China (31170623), and Agricultural Projects of Zhejiang Province (2010C12011, 2012C12908-2).

Received: 26 May 2014 Accepted: 11 August 2014

Published: 29 August 2014

\section{References}

Aggarwal RK, Hendre PS, Varshney RK, Bhat PR, Krishnakumar V, Singh L (2007) Identification, characterization and utilization of EST-derived genic microsatellite markers for genome analyses of coffee and related species. Theor Appl Genet 114(2):359-372

Akagi H, Yokozeki Y, Inagaki A, Mori K, Fujimura T (2001) Micron, a microsatellitetargeting transposable element in the rice genome. Mol Genet Genomics 266:471-480

Botstein D, White RL, Skolnick M, Davis RW (1980) Construction of a genetic linkage map in man using restriction fragment length polymorphisms. Am J Hum Genet 32:314-331

Cordeiro GM, Casu R, McIntyre CL, Manners JM, Henry RJ (2001) Microsatellite markers from sugarcane (Saccharum spp.) ESTs cross-transferable to erianthus and sorghum. Plant Sci 160(6):1115-1123

Das M, Bhattacharya S, Singh P, Filgueiras TS, Pal A (2008) Bamboo taxonomy and diversity in the era of molecular markers. In: Jean-Claude K, Michel D (eds) Advances in Botanical Research. Academic Press, San Diego, CA 92101-4495, USA, pp 225-268

Dong WJ, Wu MD, Lin Y, Tang DQ (2011) Evaluation of 15 caespitose bamboo EST-SSR markers for cross-species/genera transferability and ability to identify interspecies hybrids. Plant Breed 130:596-600

Doyle JJ, Doyle JL (1987) A rapid isolation procedure for small quantities of fresh leaf materials. Phytochem Bull 19:11-15
Feng S, Li W, Huang H, Wang J, Wu Y (2009) Development, characterization and cross-species/genera transferability of EST-SSR markers for rubber tree (Hevea brasiliensis). Mol Breed 23(1):85-97

Fu J (2001) Chinese Moso Bamboo: Its importance. Bamboo 22(5):5-7

Gui Y, Sheng W, Quan L, Zhou C, Long S, Zheng H, Jin L, Zhang X, Ma N, Fan L (2007) Genome size and sequence composition of moso bamboo: A comparative study. Sci China Series C: Life Sci 50(5):700-705

Guilherme FAG, Ressel K (2001) Biologia floral e sistema de reprodução de Merostachys riedeliana (Poaceae: Bambusoideae). Revta brasil Bot 24:205-211 (in Spanish with English abstract)

Higa JK, Liang Z, Williams PG, Panee J (2012) Phyllostachys edulis compounds inhibit palmitic acid-induced monocyte chemoattractant protein 1 (MCP-1) production. PLoS One 7(9):e45082

Huang X, Madan A (1999) CAP3: A DNA sequence assembly program. Genome Res 9:868-877

Jakupiak JP, Wells RD (1999) Genetic instabilities in (CTG.CAG) repeats occur by recombination. J Biol Chem 274:23468-23479

Janzen DH (1976) Why bamboos wait so long to flower. Ann Rev Eco Syst 7:347-391

Kitamura K, Kawahara T (2009) Clonal identification by microsatellite loci in sporadic flowering of a dwarf bamboo species, Sasa cernua. J Plant Res 122(3):299-304

Kitamura K, Kawahara T (2011) Estimation of outcrossing rates at small-scale flowering sites of the dwarf bamboo species, Sasa cernua. J Plant Res 124:683-688

Kofler R, Bartos J, Gong L, Stift G, Sucha'nkova P, Suchankova P, Simkova H, Berenyi M, Burg K, Dolezel J, Lelley T (2008) Development of microsatellite markers specific for the short arm of rye (Secale cereale L.) chromosome 1. Theor Appl Genet 117(6):915-926

Li XL, Liu S, Shong WQ, Chen RY, Wang WZ (1999) Chromosome numbers of forty species of scattered bamboo. Acta Phytotax Sinica 37(6):541-544 (in Chinese with English abstract)

Li XL, Lin RS, Fen XL, Qi ZX, Song WQ, Chen RY (2001) Chromosome numbers of ninety-four species of caespitose bamboo. Acta Phytotax Sinica 39(5):433-442 (in Chinese with English abstract)

Liang X, Chen X, Hong Y, Liu H, Zhou G, Li S, Guo B (2009) Utility of EST-derived SSR in cultivated peanut (Arachis hypogaea L.) and Arachis wild species. BMC Plant Biol 9:35

Lin XC, Ruan XS, Lou YF, Guo XQ, Fang W (2009) Genetic similarity among cultivars of Phyllostachys pubescens. Plant Syst Evol 277:67-73

Liu KJ, Muse SV (2005) PowerMarker: An integrated analysis environment for genetic marker date. Bioinformatics 21(9):2128-2212

Lu HJ, Fellers JP, Friesen TL, Meinhardt SW, Faris JD (2006) Genomic analysis and marker development for the Tsn1 locus in wheat using bin-mapped ESTs and flanking BAC contigs. Theor Appl Genet 112(6):1132-1142

Lu JJ, Yoshinaga K, Fang W, Tang DQ (2009) Identification of the bamboo hybrids by SSR markers. Sci Silv Sin 45(3):29-34 (in Chinese with English abstract)

Malysheva L, Sjakste T, Matzk F, Roder M, Ganal M (2003) Molecular cytogenetic analysis of wheat-barley hybrids using genomic in situ hybridization and barley microsatellite markers. Genome 46:314-322

Miyazaki Y, Ohnishi N, Hirayama K, Nagata J (2009) Development and characterization of polymorphic microsatellite DNA markers for Sasa senanensis (Poaceae: Bambuseae). Conserv Genet 10:585-587

Monden Y, Naito K, Okumoto Y, Saito H, Oki N, Tsukiyama T, Ideta O, Nakazaki T, Wessler SR, Tanisaka T (2009) High potential of a transposon mPing as a marker system in japonica $\times$ japonica cross in rice. DNA Res 16(2):131-140

Nayak S, Rout GR (2005) Isolation and characterization of microsatellites in Bambusa arundinacea and cross species amplification in other bamboos. Plant Breed 124:559-602

Nishiwaki A, Konno Y (1990) Pollination system in two dwarf bamboo species. Bamboo J 8:17-20

Panaud O, Chen X, McCouch SR (1996) Development of microsatellite markers and characterization of simple sequence length polymorphism (SSLP) in rice (Oryza sativa L.). Mol Gen Genet 252:597-607

Peng Z, Lu T, Li L, Liu X, Gao Z, Hu T, Yang X, Feng Q, Guan J, Weng Q, Fan D, Zhu C, Lu Y, Han B, Jiang Z (2010) Genome wide characterization of the biggest grass, bamboo, based on 10,608 putative full-length cDNA sequences. BMC Plant Biol 10:116

Rajora P, Rahman H (2003) Microsatellite DNA and RAPD fingerprinting, identification and genetic relationships of hybrid poplar (Populus $\mathrm{x}$ canadensis) cultivars. Theor Appl Genet 106:470-477 
Richard GF, Kerrest A, Dujon B (2008) Comparative genomics and molecular dynamics of DNA repeats in eukaryotes. Micr Mol Bio Rev 72:686-727

Richards RI, Sutherland GR (1994) Simple repeat DNA is not replicated simply. Nature Genet 6:114-116

Ritland K (1996) Multilocus mating system program - MLTR., Available free of charge at http://genetics.forestry.ubc.ca/ritland/programs.html

Ritland K (2002) Extensions of models for the estimation of mating systems using $n$ independent loci. Heredity 88:221-228

Ritland K, Jain SK (1981) A model for the estimation of outcrossing rate and gene frequencies using $n$ independent loci. Heredity 47:35-52

Rossetto M (2001) Sourcing of SSR markers from related plant species. In: Henry RJ (ed) Plant genotyping--the DNA fingerprinting of plant. CABI Publishing, Wallingford, Oxon OX10 8DE, UK, pp 211-224

Rossi M, Araujo PG, Paulet F, Garsmeur O, Dias VM, Chen H, Van Sluys MA, D'Hon A (2003) Genomic distribution and characterization of EST-derived resistance gene analogs (RGAs) in sugarcane. Mol Genet Genomics 269(3):406-419

Scherer S (2008) A short guide to the human genome. Cold Spring Harbor University Press, Cold Spring, NY

Sharma RK, Gupta P, Sharma V, Sood A, Mohapatra T, Ahuja PS (2008) Evaluation of rice and sugarcane SSR markers for phylogenetic and genetic diversity analyses in bamboo. Genome 51(2):91-103

Sharma V, Bhardwaj P, Kumar R, Sharma RK, Sood A, Ahuja PS (2009) Identification and cross-species amplification of EST derived SSR markers in different bamboo. Conserv Genet 10:721-724

Squirrell J, Hollingsworth PM, Woodhead M, Russell J, Lowe AJ, Gibby M, Powell W (2003) How much effort is required to isolate nuclear microsatellites from plants? Mol Ecol 12(6):1339-1348

Tang DQ (2009) Genomic sequencing and its application for biological and evolutional research in bamboo. Bamboo J 26:1-10

Tang DQ, Lu JJ, Fang W, Zhang S, Zhou MB (2010) Development, characterization and utilization of GenBank microsatellite markers in Phyllostachys pubescens and related species. Mol Breed 25:299-311

Temnykh S, DeClerck G, Lukashova A, Lipovich L, Cartinhour S, McCouch SR (2001) Computational and experimental analysis of microsatellites in rice (Oryza sativa L.): frequency, length variation, transposon associations, and genetic marker potential. Genome Res 11:1441-1452

Tero N, Neumeier H, Gudavalli R, Schlo"tterer C (2006) Silene tatarica microsatellites are frequently located in repetitive DNA. J Evol Biol 19(5):1612-1619

Thiel T, Michalek W, Varshney RK, Graner A (2003) Exploiting EST databases for the development and characterization of gene-derived SSR-markers in barley (Hordeum vulgare L.). Theor Appl Genet 106:411-422

Tomilin NV (2008) Regulation of mammalian gene expression by retroelements and non-coding tandem repeats. Bioessays 30:338-348

Watanabe M, Ueda K, Manabe I, Akai T (1982) Flowering, seeding, germination and flowering periodicity of Phyllostachys pubescens. J Jpn For Soc 64:107-111

Wei F, Wing RA, Wise RP (2002) Genome dynamics and evolution of the Mla (powdery mildew) resistance locus in barley. Plant Cell 14:1903-1917

Wu M, Dong W, Tang DQ (2009) Identification of four caespitose hybrid bamboos by using SSR markers. Mol Plant Breed 7(5):959-965 (in Chinese with English abstract)

Yang HQ, Yang JB, Gao J, Yang YM, Peng S, Li DZ (2008) A molecular phylogenetic and fruit evolutionary analysis of the major groups of the paleotropical woody bamboos (Gramineae: Bambusoideae) based on nuclear ITS, GBSSI gene and plastid trnL-F DNA sequences. Mol Phylogenet Evol 48(3):809-824

Yu JK, Dake TM, Singh S, Benscher D, Li W, Gill B, Sorrells ME (2004) Development and mapping of EST-derived simple sequence repeat markers for hexaploid wheat. Genome 47(5):805-818

Zhang LY, Bernard M, Leroy P, Feuillet C, Sourdille P (2005) High transferability of bread wheat EST-derived SSRs to other cereals. Theor Appl Genet 111(4):677-687

Zhong H, Zhou MB, Xu CM, Tang DQ (2010) Diversity and evolution of Pong-like elements in Bambusoideae subfamily. Biochem Syst Ecol 38:750-758

Zhou MB, Lu JJ, Zhong H, Liu XM, Tang DQ (2010a) Distribution and diversity of PIF-like transposable elements in the Bambusoideae subfamily. Plant Sci 179:257-266
Zhou MB, Lu JJ, Zhong H, Tang KX, Tang DQ (2010b) Distribution and polymorphism of mariner-like elements in the Bambusoideae subfamily. Plant Syst Evol 289:1-11

Zhou MB, Zhong H, Zhang QH, Tang KX, Tang DQ (2010C) Diversity and evolution of Ty1-copia retroelements in representative tribes of Bambusoideae subfamily. Genetica 138:861-868

doi:10.1186/2193-1801-3-486

Cite this article as: Lin et al:: Identification, cross-taxon transferability and application of full-length cDNA SSR markers in Phyllostachys

pubescens. SpringerPlus 2014 3:486.

\section{Submit your manuscript to a SpringerOpen ${ }^{\odot}$ journal and benefit from:}

- Convenient online submission

$\checkmark$ Rigorous peer review

- Immediate publication on acceptance

- Open access: articles freely available online

- High visibility within the field

- Retaining the copyright to your article

Submit your next manuscript at $>$ springeropen.com 